\title{
Sudden unexpected death in epilepsy in children: a focused review of incidence and risk factors
}

\author{
Anurag Saxena, ${ }_{1}^{1}$ Lliwen Jones, ${ }^{2}$ Rohit Shankar, ${ }^{3}$ Brendan McLean, ${ }_{1}^{4}$ \\ Craig G J Newman, ${ }^{5}$ Khalid Hamandi ${ }^{6}$
}

${ }^{1}$ The Noah's Ark Children's Hospital for Wales, Cardiff, UK

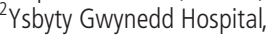
Bangor, UK

${ }^{3}$ Exeter Medical School, Exeter, UK

${ }^{4}$ The Royal Cornwall Hospitals NHS Trust, Truro, UK

${ }^{5}$ Plymouth University Peninsula Schools of Medicine and Dentistry, Plymouth, UK

${ }^{6}$ Wales Epilepsy Unit, Department of Neurology, University Hospital of Wales, Cardiff, UK

\section{Correspondence to} Dr Khalid Hamandi, Wales Epilepsy Unit, University Hospital of Wales, Cardiff, CF144XW, UK; hamandik@cf. ac.uk

Received 24 November 2017 Revised 21 February 2018

Accepted 12 March 2018 Published Online First 9 April 2018
Check for updates

To cite: Saxena A, Jones $L$, Shankar R, et al. J Neurol Neurosurg Psychiatry 2018:89:1064-1070.

\section{ABSTRACT}

Sudden unexpected death in epilepsy (SUDEP) in children, although rare, needs critical attention given the tragic nature and devastating consequences for families and caregivers. True incidence is unknown and risk factors are not completely understood, more so in children compared with adults. A focused narrative review of available studies on paediatric SUDEP was undertaken to comprehend its risk factors and to develop strategies to recognise and where possible modify SUDEP risk and ultimately reduce incidence. We reviewed 16 population-based studies from various settings. We found overlapping risk factors from different studies. The prime risk factor is uncontrolled seizures. This review supports the view that children entering adolescence with optimal seizure control could be a key aspect in reducing adult mortality related to SUDEP. Ideally, clinicians would want to be able to predict prospective, individualised SUDEP risk, which is challenging due to a myriad of risk factors and an inherent non-homogeneous paediatric epilepsy population. Nevertheless, an adequate evidence base exists as evidenced by this review to support information giving and communication to support young people with epilepsy and their families in being active partners in recognising and reducing their SUDEP risk. More work particularly in the form of prospective studies and registries are needed to further clarify true incidence which may have been previously underestimated and to update risk factors.

\section{INTRODUCTION}

Epilepsy is a common neurological disease in children. Population studies give an incidence from 41 to 187 per 100000 person-years, ${ }^{1}$ highest in the first year of life and declining to the same rate as adults by the end of the first decade. The wide range in reported incidence arises as a result of differences in study populations sampled, diagnostic criteria, search methods and age bands examined.

Paediatric sudden unexpected death in epilepsy (SUDEP), although rare, is a tragic and catastrophic event, with far-reaching effects on family, carers and wider society that deserves full attention, investigation and prevention. The incidence of SUDEP in children is typically reported as five times less than that seen in adults, varying between 1.1 and 3.4 per 10000 person-years (PYs), ${ }^{2-4}$ in contrast to SUDEP rates in adults of 1 to 9 per 1000 PYs. ${ }^{5}$ However, a recent nationwide population-based study challenges these figures, finding similar rates of SUDEP in children and adults. ${ }^{7}$ The currently perceived lower incidence of SUDEP in children may, in part, be due to under-reporting of SUDEP cases, and be responsible for less awareness of SUDEP in paediatric healthcare professionals and hence less emphasis on imparting SUDEP information in paediatric practice. ${ }^{18-11}$ The paucity of information around childhood SUDEP may result in clinician discomfort when providing SUDEP information and parental education on mitigation of this condition. ${ }^{12} 13$ This situation may lead to less informed and less prepared families. ${ }^{14}$

Risk factors are now well recognised for SUDEP in adults ${ }^{1516}$ and are of value in sharing information and hopefully modifying SUDEP risk. A series of potentially modifiable risk factors have been identified that can be used as part of a structured risk assessment and information giving in adults. ${ }^{17}$ SUDEP risks are not as well characterised in children. We, therefore, conducted a review of the literature of SUDEP in children to identify risk factors that could be better used in imparting information and management of children with epilepsy.

\section{METHODS}

We conducted a detailed literature review in Medline through PubMed using following keywords: Children (OR Child OR Childhood OR Paediatric) AND (Epilepsy OR Seizure OR Seizures OR Epileptic seizures OR Epileptic encephalopathy) AND (SUDEP OR Sudden unexpected death in epilepsy OR Sudden unexplained death in epilepsy) from 1995 to February 2017.

SUDEP historically has been given two complementary definitions. ${ }^{18} 19$ Nashef proposed 'Sudden, unexpected, witnessed or unwitnessed, nontraumatic and non-drowning death in patients with epilepsy, with or without evidence for a seizure and excluding documented status epilepticus, in which postmortem examination does not reveal a toxicologic or anatomic cause of death'. ${ }^{19}$ Annegers used similar criteria but did not specifically exclude death following status epilepticus and proposed definite, probable and possible categories based on available evidence, for example, postmortem data and other circumstantial data. ${ }^{18}$ To try and overcome some of these difficulties, a unifying definition incorporating both the definitions was proposed recently. ${ }^{20}$ In the articles reviewed here, we have indicated where available the SUDEP definitions applied, but recognise that SUDEP data in retrospective case series may be incomplete. ${ }^{21}$ 
Three of the authors (AS, LJ and $\mathrm{KH}$ ) independently reviewed titles and abstracts. The publications were categorised to 'SUDEP and risk factors', 'case reports', 'information and attitudes', 'reviews', 'putative mechanisms', 'seizure monitoring' and 'other' (reports in adult populations, basic science/animal studies not directly related to SUDEP mechanisms, commentries, etc). This last group included publications where childhood SUDEP was not the main focus of the article and included studies in adults, epidemiological studies without narrative accounts or not addressing epilepsy-related deaths per se. A consensus was reached to limit our analysis to original research with population studies on 'SUDEP and risk factors'.

\section{RESULTS}

We identified 275 studies with our initial search of which 16 population-based studies were selected as 'SUDEP and risk factors' for further review; two of these were subsequently excluded, after accessing the full article as outside the scope of this review, one case report ${ }^{22}$ and one a study of febrile seizure-related mortality. ${ }^{23}$ Three additional studies were included as of particular interest, two population-based studies published after the literature search cut-off after notification alert of publication to one of the authors, ${ }^{24}$ and during peer review ${ }^{7}$ and one mechanistic study, ${ }^{2}$ providing a total of 16 population risk factor articles for review (table 1$)^{25-36}$ and one mechanistic study. The breakdown from the original search was as follows: case reports $(n=14)$, information and attitudes $(n=20)$, reviews $(n=29)$, putative mechanisms $(n=74)$, seizure monitoring $(n=10)$ and other $(\mathrm{n}=112)$.

Nashef et al reported the first study examining the incidence and characteristics of SUDEP in a group of children and adolescents attending a special residential school. ${ }^{25}$ Most had severe epilepsy, with weekly or daily seizures. Coroners reports, postmortem findings, school records and death certificates were used to compile information surrounding deaths. Twenty-eight deaths were identified in 310 pupils, with a mean age at death of 19 (9-32) years. Fourteen cases were classified as SUDEP. Four were under 17 (ages 10, 12, 14 and 16). All 14 had a history of generalised tonic-clonic seizures (GTCS). Ten of 14 were found dead in bed or the bedroom floor in the morning. None of the SUDEP cases occurred while under supervision at school (which had overnight sound monitoring and staff attending seizures) but occurred either after pupils had left school $(n=12)$ or when on leave $(n=2)$.

Donner et al identified 27 SUDEP cases from three sources. ${ }^{26}$ Nineteen cases were from the Ontario Chief Coroner's office, four identified from the Ontario Paediatric Forensic Pathology Unit and four additional cases were reported by the staff of the Division of Neurology at the Hospital for Sick Children, Toronto, Canada. Seventeen (63\%) of the SUDEP cases were boys. Only cases with postmortem reports were considered to be definite SUDEP $(n=17)$. Age at death ranged from 8 months to 15 years. Fourteen SUDEP cases had symptomatic epilepsy (52\%), five had cryptogenic epilepsy (18\%) and eight had idiopathic epilepsy (30\%). In 23 SUDEP cases, where information on seizure type was available $(n=23)$, all had a history of GTCS. Antiepileptic drug (AED) history was available in 26 children: 12 children $(46 \%)$ were on one AED, 10 (38\%) were on two AEDs and 3 $(12 \%)$ were on three AEDs. Seven children (35\%) had one or more AEDs below the therapeutic range, and 12 children (60\%) had AEDs within the therapeutic range. Sixteen (59\%) reported SUDEP cases were found in bed. Ten children had witnessed deaths. All 10 were seen to have a loss of consciousness followed by cardiorespiratory arrest, 5 of which had a preceding seizure, but in the other 5 , there was no preceding seizure. This study is one of only three reports, where age at death was entirely in the paediatric population, whereas the other 11 studies also considered adolescent and adult deaths (see table 1).

Camfield et al reported a population-based cohort study linking marriage and death records to a comprehensive database of children diagnosed with epilepsy. ${ }^{1}$ The study examined death certificates, postmortem reports and physician records of children who had died. Families of these children were contacted to see if SUDEP could have occurred. Twenty-six deaths were identified from 692 children with epilepsy, 22 were reported as not unexpected and occurred in those with a severe neurological deficit. In these 22, most deaths were due to aspiration pneumonia or sepsis, the median age of mortality in this group was 10 (1-29) years. Four deaths were unexpected, and all were young adults aged 18-30 years with only one reported as SUDEP, a woman with tuberous sclerosis and poorly controlled seizures. While there were no paediatric deaths, the study is still included here in that the title and aims were to study 'death in children with epilepsy'. Furthermore, it is notable that in this retrospective study of mortality records the higher rate of death in children was reported as due to functional deficits of comorbid neurological disorders and no case of SUDEP in childhood was reported.

McGregor et al reviewed the clinical data from all childhood-onset epilepsy SUDEP cases over 12 -year period. ${ }^{27}$ Deaths were identified following parent reporting, or by contact from a local clinician or the county medical examiner. The study identified 17 cases of SUDEP (classified as 7 definite; 9 probable; 1 possible). Nine were under the age of 16 at the time of death. Postmortem reports were available in seven cases. The age at death ranged from 1.8 to 25.9 years. Twelve of the SUDEP cases $(71 \%)$ had structural lesions, cognitive delay or both. All patients had GTCS (with symptomatic focal epilepsy). One child with febrile seizures died of SUDEP at 1 year and 10 months of age. Thirteen of 17 died in sleep, and 5 of 17 were found prone; in all others, the location and position of death were not known.

A study from the Netherlands, by Vlooswijk et al, identified 274 deaths in 4400 patients treated during the study period with 5 definite SUDEP, 24 probable SUDEP and 21 possible SUDEP cases. ${ }^{28}$ Seven were aged 16 or younger. Two of these were classified as definite SUDEP, one aged 7 was hospitalised with tonsillitis and found dead in bed at 03:20, the other had seizures with apnoeas and had a witnessed apnoea. Of the other five probable childhood SUDEP cases, three were found dead in bed, one found at home, and one not known. In a statistical comparison between all SUDEP and non-SUDEP cases of epilepsy deaths, no correlation was found with gender, type of seizure, seizure frequency, comorbid conditions, medications, particular AED, learning difficulty or psychotropic medication.

Terra et al identified 12 SUDEP cases from 835 patient records evaluated. ${ }^{29}$. All SUDEP cases were aged 17 or under (median 7; range 2-17). The authors did not give any account of the circumstances of death, detail of epilepsy diagnosis or aetiology, seizure type or medical treatment. Seizure frequency was reported, with six having daily seizures, three had weekly seizures, one had 1 seizure per month and two had 2-4 seizures per year.

A database review for definite or probable SUDEP in children found four cases of SUDEP in children (aged 4.2, 4.2, 4.3 and 11.1 years, respectively) from a hospital registry. ${ }^{30}$ There was one definite and three probable SUDEP cases. Two children were found dead in bed, and in two cases the death was witnessed. In both observed cases, the child stopped breathing without signs of 


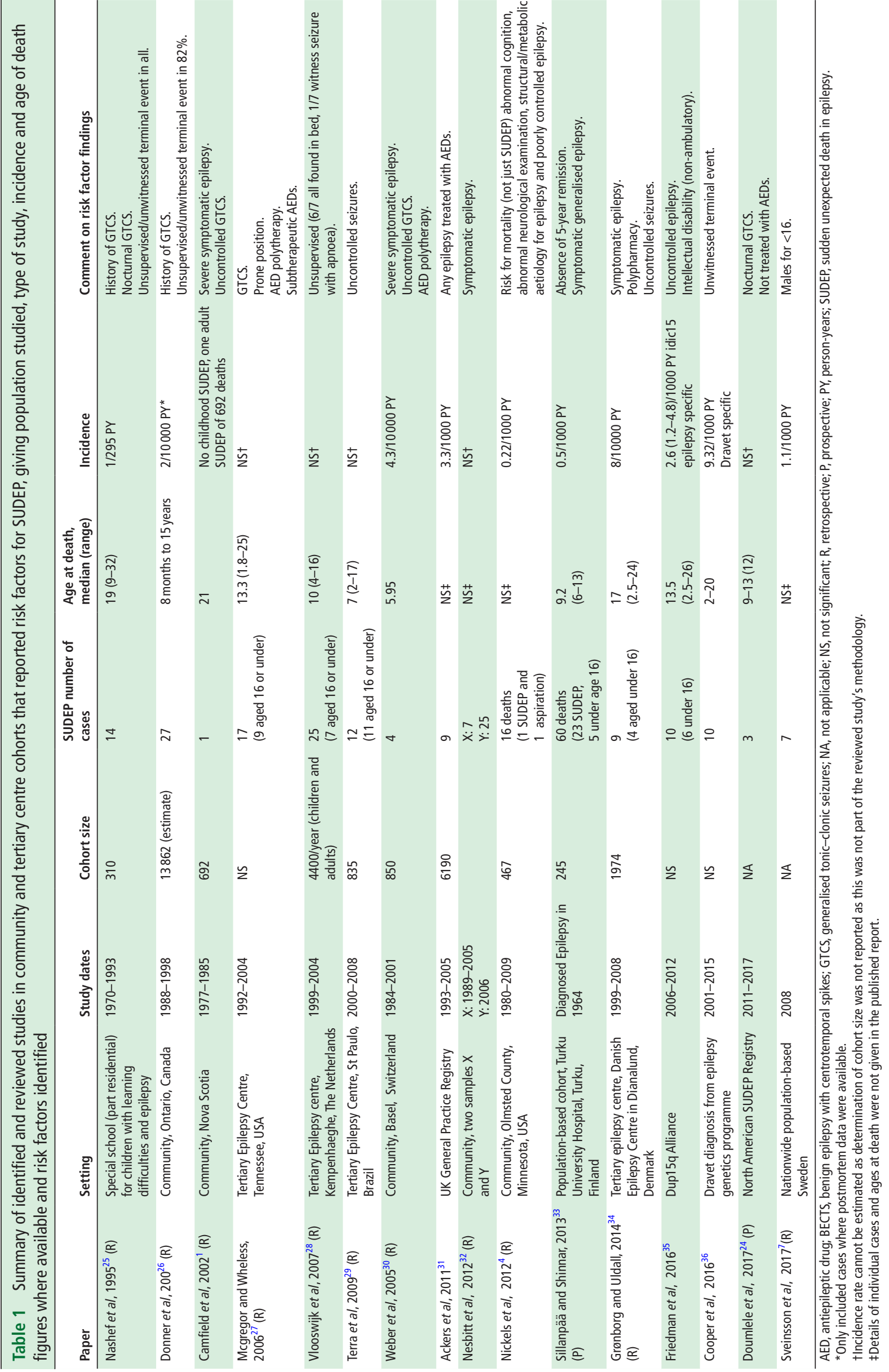


a convulsive seizure. Both cases had a severe learning difficulty, and one had a history of previous apnoeic attacks. All SUDEP cases had the onset of epilepsy in infancy, three cases had daily seizures and one had seizure clusters. The study only included those with an 'inherited' rather than 'acquired' epilepsy (for example, excluded those with epilepsy following brain injury) thus introducing bias in the type, incidence and age of onset of epilepsy and possible cofactors for SUDEP.

A retrospective cohort study by Ackers et al aimed to identify cases and causes of the death in children prescribed AEDs. ${ }^{31}$ Details of seizure type or frequency and circumstances of death were not given. A consensus panel of experts undertook causality assessment for the likelihood of association between the AEDs and death. From 6190 children there were 151 deaths, $60 \%$ had a structural or metabolic cause for their epilepsy and unknown in $22 \%$. Death was attributable to epilepsy in 18 cases, with 9 cases of SUDEP-5 probable SUDEP, 4 possible SUDEP-equating to an incidence of 3.3/10 000 PYs. Four of the SUDEP cases had no other known neurological disorder, and three children were on AED monotherapy, three of the SUDEP cases were arrived at after the expert panel over-ruled the death certificate cause of death. The circumstances of death and possible risks were not presented.

Nesbitt $e$ t al analysed two large databases of deaths in children with epilepsy looked at their clinical details and death certificates retrospectively. ${ }^{32}$ The combined data from the two sources gave 265 deaths in children with epilepsy further broken down as 97 deaths in the first sample and 168 deaths in the second sample. The first sample had a bias towards children with more severe epilepsy but had greater clinical detail, while the second sample had a well-defined ascertainment method, but clinical data were limited. In 32 childhood deaths, the cause remained unexplained after detailed analysis. All the unexplained deaths were in children with symptomatic epilepsy. No SUDEP cases were recorded in either of the datasets, though SUDEP definitions (possible, probable, definite) were not applied, and in 116 of 372 deaths, the contribution of epilepsy to death was reported as unascertained.

A retrospective review of all children aged between 1 month and 17 years was done by Nickels et al. ${ }^{4} \mathrm{~A}$ total of 467 children with epilepsy had their data analysed with follow-up of around 30 years, amounting to 4500 PYs. Sixteen of 467 children died over the follow-up period. The mortality rate was $3.5 / 1000$ PYs, 10 times higher than the expected national mortality rate for children. Two deaths were epilepsy related, one probable SUDEP, the other aspiration during a seizure. Significant risk factors for death in epilepsy (rather than SUDEP per se) were of an abnormal neurological examination, abnormal cognitive function, previous status epilepticus, structural/metabolic aetiology, taking more than two AEDs, and intractable epilepsy and seizures more often than every 6 months.

A report on the mortality and causes of death in 1974 patients, with childhood-onset epilepsy at a tertiary epilepsy centre, was compiled by Grønborg et al. ${ }^{34}$ The authors identified childhood deaths as published in the Danish civil register by the 1 June 2009. Relevant medical information regarding the circumstances and causes of death were derived from death certificates, postmortem reports and the medical records of terminal caregiving hospitals and the Epilepsy Centre. Nine patients $(0.45 \%$ of the study cohort) died with definite or probable SUDEP corresponding to eight SUDEP cases per 10000 patient-years. Eight of the nine patients with SUDEP had drug-resistant epilepsy. All had GTCS, and none were seizure free. All except one had symptomatic or cryptogenic epilepsy. Median age at death was 17 years (range: $2.5-24$ years). Four patients died under the age of 16 years. All childhood SUDEP cases had drug-resistant seizures, two or more AEDs and symptomatic or cryptogenic epilepsy.

Sillanpää et al published a report on a 40 -year, prospective, population-based study of childhood-onset epilepsy. ${ }^{33}$ There were 60 reported deaths, giving a standardised mortality ratio of 6.4 (95\% CI: 5.9 to 7.0$)$, 33 (55\%) were epilepsy-related. These included 23 cases of SUDEP, of which 17 definite SUDEP and 6 probable SUDEP. Five SUDEP cases were under 16 at the age of death, four with symptomatic epilepsy from perinatal complications and associated neurological disability and one with juvenile absence epilepsy who died at the age of 13 .

We considered two further studies that evaluated SUDEP in specific severe childhood epilepsy syndromes, one in the isodicentric chromosome 15 (idic15 or Dup15q) syndromes and the other in Dravet syndrome (DS).

Duplications of chromosome 15q11.2q13 affect approximately 1 in 30000 births. Clinical features of the idic 15 syndromes include early-onset, refractory epilepsy multiple seizure types, hypotonia, motor delays, learning disability and autism. Freidman et al used a retrospective case-control design. ${ }^{35}$ Twenty-one deaths among patients with the dup $15 \mathrm{q}$ syndrome in the patient registry held by the Dup $15 \mathrm{q}$ Alliance were reported between April 2006 and June 2012. Nineteen of these deaths occurred in patients with the idic15 syndrome, and two occurred in individuals with related conditions which differ cytogenetically and phenotypically. There were five definite, three probable and two possible SUDEP-related deaths, of which six were aged under 16. There was no difference in age or sex between the cases and controls. No apparent difference was seen between SUDEP cases and control subjects in the age of epilepsy onset, duration of epilepsy, male sex, having nocturnal supervision, or using a remote audio/video monitoring device. A higher proportion of SUDEP cases were non-ambulatory (OR 16.7, 95\% CI: 1.38 to 547). None of the SUDEP cases was seizure-free for over 6 months at the time of death, whereas four of the control cases $(19.0 \%)$ were seizure-free at the time of interview.

A study of mortality and SUDEP rate in DS $^{36}$ reported on 100 unrelated participants recruited to the Epilepsy Genetics Research Programme with the typical electroclinical phenotype of DS. ${ }^{37}$ Eighty-seven patients in the series had SCN1A mutations. Analysis began from either February 2001 or when the child turned 1 year of age, whichever was later, to February 2015. SUDEP was the most common cause of mortality occurring in 10/17 (59\%) deaths, between ages of 2 and 20 years. Two probable SUDEP cases were age $>18$ years at death. SUDEP was definite in three and probable in six cases. A Dravet-specific SUDEP rate of 9.32 per 1000 PYs was ascertained along with a mortality rate of 15.84 per 1000 PYs.

SUDEP in benign epilepsy with centrotemporal spikes (BECTS) from the the North American SUDEP Registry (NASR) found three cases. ${ }^{24}$ The NASR was established in 2011 and includes medical records, investigation results and interviews with family members in patients with epilepsy who died suddenly without other identifiable causes of death. Three boys aged 9-13 (median 12) with a diagnosis of BECTS were identified from 189 NASR cases, 2 were definite SUDEP and 1 probable. Independent review of the individual records confirmed typical electroclinical feature of BECTS and SUDEP, and none of these cases were treated with AEDs. All were found dead in bed, in the morning in the prone position, two with early rigour mortis suggesting a time of death before midnight and other evidence of a seizure, incontinence and tongue biting. While SUDEP in BECTS is rare, the authors suggested the prevalence could be 
1 death in 1000 . It is an important consideration when giving information to parents and families and considering treatment, particularly in the presence of GTCS. This report fell outside the initial search dates, but makes a significant contribution to the literature, reporting SUDEP in the 'idiopathic' epilepsies in a prospective registry and is thus included.

Most studies reviewed here supported a prevailing view that SUDEP risk is lower in children compared with adults. A recent study by Sveinsson et $\mathrm{al}^{7}$ challenges this view. They conducted a nationwide population-based cohort study reviewing 57775 patients diagnosed with epilepsy between 1998 and 2005 alive on 1 January 2008 and found that 1890 died (3.3\%) during 2008, 99 met the Annegers SUDEP criteria and SUDEP accounted for $5.2 \%$ of all deaths. The incidence of definite/probable SUDEP was $1.20 / 1000$ PYs and higher in men (1.41/1000 PYs) than in women $(0.96 / 1000$ PYs). All SUDEP cases $<16$ years were in boys. Most importantly, this study found that SUDEP incidence at ages $<16,16-50$ and $>50$ years was $1.11,1.13$ and 1.29, respectively, per 1000 PYs. ${ }^{7}$ Furthermore, the authors found that SUDEP was listed as the immediate cause of death in only 1 of the 99 definite, probable or possibly SUDEP cases and epilepsy was mentioned on the death certificate in only 62 of the 99 (63\%) SUDEP cases. ${ }^{7}$ The findings of this study suggest that SUDEP risk is no different across ages and highlights the potential for underestimating SUDEP incidence when relying only on death certificates to identify cases.

Lastly, the study by Freitas et $a l^{2}$ whilst not a population-based SUDEP incidence study, is included here as it proposes difference in seizure semiology between adults and children. Ictal video-electroencephalography (EEG) studies of adults and paediatric population from the Epilepsy Monitoring Unit at Rainbow Babies and Children's Hospital (children) and University Hospitals Case Medical Centre, Cleveland, Ohio, USA (adults) were reviewed. Durations of clinical tonic, clonic, total seizure features, neurophysiological postictal EEG suppression (PGES), and recovery phases, and cardiac heart rate variability measures were determined. Seizure end was defined as cessation of all clinical manifestations and/or EEG paroxysmal activities. PGES was defined as the immediate postictal (within $30 \mathrm{~s}$ ), the generalised absence of EEG activity $>10 \mu \mathrm{V}$ in amplitude. 105 seizures were studied from 12 children with 23 seizure events and 49 adults with 82 seizure events. The total seizure, tonic phase, PGES, and recovery phase durations were all found to be significantly shorter in children. PGES was present in 13/23 (57\%) GTCS events in 5/12 (42\%) paediatric patients, whereas it was present in $77 / 82$ (94\%) seizure events in $44 / 49$ (90\%) adult patients. The data from this study points to an identifiable electroclinical difference between the adult and paediatric population which may at least, in part, explain differences in SUDEP incidence.

As a result of our review, we propose the following factors influencing SUDEP risk in children:

A. Aetiology-related factors

i. Comorbidities especially cardiac or respiratory.

ii. Genetic syndromes such as SCN1a and $15 \mathrm{qDuplication}$, presenting with refractory epilepsy.

iii. Intellectual disability.

iv. MRI and EEG findings that support a diagnosis of a structural/metabolic (symptomatic) or unknown (cryptogenic) epilepsy.

B. Environmental factors

i. Night-time surveillance and intervention.

ii. Identified caregiver.

C. Seizure factors i. Seizure frequency and severity.

ii. Uncontrolled seizures, especially nocturnal GTCS, even at low frequency.

iii. Failure of 5-year terminal remission.

D. Treatment factors (most studies lacked sufficient data to test for or identify statistically significant risk. However, this remains an important consideration regarding seizure control, particularly nocturnal GTCS)

i. AED treatment.

ii. Polypharmacy.

iii. Adherence and delivery.

E. Other

i. Change in physical health status-Acute changes in physical health such as infections and fever in those who already have a history of seizures could further accentuate risk. Empirical evidence to establish a direct association to SUDEP is unavailable; however, logic dictates that this needs to be considered in any change in seizure patterns for the worse.

\section{DISCUSSION}

The review has shown that there is heterogeneity in studies both regarding definition ${ }^{18}{ }^{19}$ used for SUDEP, study setting and sources of data: community based $(n=5)$, special school population $(n=1)$, tertiary epilepsy centres $(n=4)$ and population or national patient registers $(n=6)$. Despite these differences common themes, uncontrolled epilepsy, symptomatic epilepsy, lack of supervision and polypharmacy emerge. Another noteworthy feature, which is apparent, is that SUDEP rate in adolescents, likely to still be under the care of paediatricians or undergoing transition, even in those studies that report a lower SUDEP rate in children approaches that of the adult population with epilepsy. We found that studies reporting on paediatric SUDEP have a wide diversity in the methods employed, population studied and definition of SUDEP used. As such, given this range of factors, a meta-analysis or a systematic review is not currently possible. A meta-analysis aims to pool data from conceptually similar studies on the assumption that greater statistical power can be gained by minimising the error from smaller studies, and we did not feel this could be done on current SUDEP studies given the range of differences in population and, methods, hence proceeded with this narrative review.

Mortality in children with epilepsy is significantly higher than the general population. Most of the deaths were related to underlying neurological conditions rather than epilepsy. ${ }^{38}$ Epidemiological studies of SUDEP in children were mostly retrospective. There are recognised difficulties with case ascertainment in retrospective studies due to the lack of SUDEP awareness. Databases may be incomplete, a postmortem lacking or interpreted differently and other competing comorbidities or aetiologies may confuse the cause of death. Not all cases are captured through databases; for example, Donner et $a l^{26}$ found eight additional cases from an alternative source, using data from the Ontario Chief Coroner's office. As a consequence, prospective or casecontrol studies give a higher incidence of SUDEP than retrospective epidemiological studies. Another confound is that while studies report 'SUDEP in childhood epilepsy', more detailed review of articles finds that many refer to childhood-onset epilepsy, but include deaths across all ages, including adults, thus making a clear separation in incidence rates and risk factors in a paediatric group more difficult. Some degree of age stratification is needed, and we suggest to reflect differences in aetiological risk and brain development and maturation, for example, $<2$, 
$2-10,10-14,14-18$ and $>18$ year age groups. Stratifying the risk factors in such age groups would be better to inform families as well as clinicians.

Another finding was that sudden death in children with severe neurodisability and refractory epilepsy is sometimes considered 'expected'. 32 This is another potential source of under-reporting of SUDEP. Any seizure-related death, where the mechanism of death is intrinsically related to a seizure and/or the absence of other identifiable cause of death should be reported as SUDEP, regardless of the underlying epilepsy aetiology and without undue emphasis or judgement on the word 'unexpected'. The term 'SUDEP plus' proposed by Nashef et al ${ }^{20}$ would capture such cases, where a pre-existing condition could have contributed to an epilepsy death. ${ }^{20}$ At the other end of the seizure severity spectrum, it is important to recognise SUDEP in children with a relatively 'mild' epilepsy, such as BECTS. ${ }^{24}$ It is notable that, the SUDEP cases in BECTS reported in the study by Doumlele et al ${ }^{24}$ occurred in those children not treated with AEDs.

Although childhood SUDEP risk is highest for patients with epilepsy not in remission, even in otherwise neurologically intact children, this risk may not manifest until adolescence and adulthood. ${ }^{33}$ This circumstance may result in paediatric epileptologists 'being lulled into a false sense of security' as they have fewer direct experiences of SUDEP in patients under their care. ${ }^{33}$ This finding highlights the importance of SUDEP awareness and imparting information for all ages. Young adults may start living independently away from parental supervision, have increased risk-taking behaviour with the use of alcohol and recreational drugs in addition to non-adherence to AEDs and sleep deprivation. These reasons, as well as changes in seizure semiology-related factors, ${ }^{2}$ lead to adolescents being vulnerable to increase SUDEP risk.

Underlying mechanisms in SUDEP are beyond the scope of this review but appear to point towards a combination of brainstem-mediated, central, postictal apnoea and cardiac mechanisms. ${ }^{39}$ There may be mechanistic differences related to seizure semiology in SUDEP in children compared with adults. The tonic phase of GTCS - and hence brainstem and respiratory centre involvement-and PGES is shorter as compared with adults. ${ }^{2}$ It has been proposed that the change in seizure semiology and related autonomic changes, along with an increase in PGES duration, in adolescence and adulthood may also contribute to changes in SUDEP risk with age. ${ }^{240}$ Further research is needed to confirm these findings. There are similarities in being found in a prone position in cases of SUDEP ${ }^{27}$ and sudden infant death syndrome (SIDS). Indeed, the advice of 'Back to Sleep' (now called 'Safe to Sleep') has been effective in reducing the SIDS mortality, ${ }^{41}$ and it becomes impractical after 6 months of age when babies tend to move around while asleep.

\section{CONCLUSION}

Currently, SUDEP prevention strategies are based on identifying and addressing modifiable risk factors. It is an area that has now been well studied in adult populations, ${ }^{42}$ but remains relatively unexplored in paediatrics. We highlight key risk factors in this review and a need for better recording of SUDEP in death certificates alongside prospective registries for more accurate estimates of incidence and risk factors.

Contributors $\mathrm{RS}, \mathrm{KH}, \mathrm{BM}$ conceived the paper. $\mathrm{KH}$ and $\mathrm{LJ}$ developed and conducted the literature search. KH, LJ and AS screened and selected the papers. AS and $\mathrm{KH}$ drafted the paper. All authors reviewed and contributed to the manuscript and approved the final version.
Funding This research received no specific grant from any funding agency in the public, commercial or not-for-profit sectors.

Competing interests RS is a major stakeholder of the 'SUDEP and Seizure Safety Checklist'. RS, BM and CGJN are developers and key stakeholders of EpSMon. RS has received institutional and research support and personal fees from LivaNova, UCB, Eisai, Special Products, Bial and Desitin outside the submitted work. BM has received research support and personal fees from Eisai, UCB and Desitin outside the submitted work. KH has received institutional and personal, consultancy and speaker fees from Eisai, UCB and Bial, outside this submitted work.

\section{Patient consent Not required.}

Provenance and peer review Not commissioned; externally peer reviewed.

(c) Article author(s) (or their employer(s) unless otherwise stated in the text of the article) 2018. All rights reserved. No commercial use is permitted unless otherwise expressly granted.

\section{REFERENCES}

1 Camfield CS, Camfield PR, Veugelers PJ. Death in children with epilepsy: a populationbased study. Lancet 2002;359:1891-5.

2 Freitas J, Kaur G, Fernandez GB, et al. Age-specific periictal electroclinical features of generalized tonic-clonic seizures and potential risk of sudden unexpected death in epilepsy (SUDEP). Epilepsy Behav 2013;29:289-94.

3 Milroy CM. Sudden unexpected death in epilepsy in childhood. Forensic Sci Med Pathol 2011;7:336-40.

4 Nickels KC, Grossardt BR, Wirrell EC. Epilepsy-related mortality is low in children: a 30-year population-based study in Olmsted County, MN. Epilepsia 2012;53:2164-71.

5 Nashef L, Fish DR, Sander JW, et al. Incidence of sudden unexpected death in an adult outpatient cohort with epilepsy at a tertiary referral centre. J Neurol Neurosurg Psychiatry 1995; 58:462-4

6 Shorvon S, Tomson T. Sudden unexpected death in epilepsy. Lancet 2011;378:2028-38.

7 Sveinsson 0, Andersson T, Carlsson S, et al. The incidence of SUDEP: a nationwide population-based cohort study. Neurology 2017;89:170-7.

8 Berl MM, Goodkin HP, Kroner BL, et al. Sudden death in epilepsy: knowledge among pediatric providers. J Pediatr 2017;188:291-3.

9 RamachandranNair R, Jack SM. SUDEP: What do adult patients want to know? Epilepsy Behav 2016:64:195-9.

10 Ramachandrannair R, Jack SM, Meaney BF, et al. SUDEP: what do parents want to know? Epilepsy Behav 2013:29:560-4.

11 Stevenson MJ, Stanton TF. Knowing the risk of SUDEP: two family's perspectives and the danny did foundation. Epilepsia 2014;55:1495-500.

12 Morse AM, Kothare SV. Pediatric sudden unexpected death in Epilepsy. Pediatr Neurol 2016:57:7-16.

13 Strzelczyk A, Zschebek G, Bauer S, et al. Predictors of and attitudes toward counseling about SUDEP and other epilepsy risk factors among Austrian, German, and Swiss neurologists and neuropediatricians. Epilepsia 2016;57:612-20.

14 Jones L, Naude JTW. Sudden unexpected death in epilepsy information provision to parents of children with epilepsy-a service evaluation. Journal of Neurology, Neurosurgery \& Psychiatry 2013;84:e2.3-e2

15 Hesdorffer DC, Tomson T, Benn E, et al. Combined analysis of risk factors for SUDEP. Epilepsia 2011;52:1150-9.

16 Tomson T, Surges R, Delamont R, et al. Who to target in sudden unexpected death in epilepsy prevention and how? Risk factors, biomarkers, and intervention study designs. Epilepsia 2016;57:4-16.

17 Shankar R, Walker M, McLean B, et al. Steps to prevent SUDEP: the validity of risk factors in the SUDEP and seizure safety checklist: a case control study. J Neurol 2016;263:1840-6.

18 Annegers JF. United States perspective on definitions and classifications. Epilepsia 1997:38:S9-S12.

19 Nashef L. Sudden unexpected death in epilepsy: terminology and definitions. Epilepsia 1997;38:S6-S8.

20 Nashef L, So EL, Ryvlin P, et al. Unifying the definitions of sudden unexpected death in epilepsy. Epilepsia 2012;53:227-33.

21 Appleton RE, Sudden ARE. Sudden, unexpected death in epilepsy in children. Seizure 1997;6:175-7.

22 Yang YJ, Yao X, Guo J, et al. Interstitial deletion 5q14.3q21.3 associated with lethal epilepsy. Am J Med Genet A 2015;167A:866-71.

23 Hesdorffer DC, Crandall LA, Friedman D, et al. Sudden unexplained death in childhood: a comparison of cases with and without a febrile seizure history. Epilepsia 2015:56:1294-300.

24 Doumlele K, Friedman D, Buchhalter J, et al. Sudden unexpected death in epilepsy among patients with benign childhood epilepsy with centrotemporal spikes. JAMA Neurol 2017;74:645-9.

25 Nashef L, Fish DR, Garner S, et al. Sudden death in epilepsy: a study of incidence in a young cohort with epilepsy and learning difficulty. Epilepsia 1995;36:1187-94 
26 Donner EJ, Smith CR, Snead OC. Sudden unexplained death in children with epilepsy. Neurology 2001;57:430-4

27 McGregor A, Wheless J. Pediatric experience with sudden unexplained death in epilepsy at a tertiary epilepsy center. J Child Neurol 2006;21:782-7.

28 Vlooswijk MC, Majoie HJ, De Krom MC, et al. SUDEP in the Netherlands: a retrospective study in a tertiary referral center. Seizure 2007;16:153-9.

29 Terra VC, Scorza FA, Sakamoto AC, et al. Does sudden unexpected death in children with epilepsy occur more frequently in those with high seizure frequency? Arq Neuropsiquiatr 2009;67:1001-2.

30 Weber P, Bubl R, Blauenstein U, et al. Sudden unexplained death in children with epilepsy: a cohort study with an eighteen-year follow-up. Acta Paediatr 2005;94:564-7.

31 Ackers R, Besag FM, Hughes E, et al. Mortality rates and causes of death in children with epilepsy prescribed antiepileptic drugs: a retrospective cohort study using the UK general practice research database. Drug Saf 2011;34:403-13.

32 Nesbitt V, Kirkpatrick M, Pearson G, et al. Risk and causes of death in children with a seizure disorder. Dev Med Child Neurol 2012;54:612-7.

33 Sillanpää M, Shinnar S. SUDEP and other causes of mortality in childhood-onset epilepsy. Epilepsy Behav 2013;28:249-55.
34 Grønborg S, Uldall P. Mortality and causes of death in children referred to a tertiary epilepsy center. Eur J Paediatr Neurol 2014;18:66-71.

35 Friedman D, Thaler A, Thaler J, et al. Mortality in isodicentric chromosome 15 syndrome: The role of SUDEP. Epilepsy Behav 2016:61:1-5.

36 Cooper MS, Mcintosh A, Crompton DE, et al. Mortality in dravet syndrome. Epilepsy Res 2016:128:43-7.

37 Shmuely S, Sisodiya SM, Gunning WB, et al. Mortality in dravet syndrome: a review. Epilepsy Behav 2016;64:69-74.

38 Berg AT, Nickels K, Wirrell EC, et al. Mortality risks in new-onset childhood epilepsy. Pediatrics 2013;132:124-31.

39 Devinsky 0, Hesdorffer DC, Thurman DJ, et al. Sudden unexpected death in epilepsy: epidemiology, mechanisms, and prevention. Lancet Neurol 2016;15:1075-88.

40 Sarkis RA, Thome-Souza S, Poh MZ, et al. Autonomic changes following generalized tonic clonic seizures: An analysis of adult and pediatric patients with epilepsy. Epilepsy Res 2015:115:113-8.

41 Hauck FR, Tanabe KO. Beyond 'Back to Sleep' : ways to further reduce the risk of sudden infant death syndrome. Pediatr Ann 2017;46:e284-90.

42 Shankar R, Donner EJ, McLean B, et al. Sudden unexpected death in epilepsy (SUDEP): what every neurologist should know. Epileptic Disord 2017:19:1-9. 